\title{
Quasi 3D Finite Element Algorithm for Rotating Mixing Flows
}

\author{
HAMEER ABRO*, AHSANULLAH BALOCH*, HAMEEDULLAH KAZI*, AND \\ MUHAMMAD ALI NIZAMANI*
}

RECEIVED ON 15.02.2017 ACCEPTED ON 17.08.2018

\begin{abstract}
The present research article presents numerical simulations of rotating of Newtonian fluid mixing flows in a cylindrical container through single rotating stirrer with agitator, where stirrer is located on the lid of container in concentric position. For this purpose a Quasi 3D (Three-Dimensional) FEA (Finite Element Algorithm) has been developed. The numerical algorithm is based on fractional stages semiimplicit Taylor-Galerkin/Pressure-Correction scheme. The simulation has been carried out to analyze the effects of agitator on mixing behavior. The numerical results show that Quasi 3D FEA is an accurate mathematical tool and able to achieve good results for flow structure in laminar regime.
\end{abstract}

Key Words: Rotating Mixing Through Stirrer with Agitator, Quasi-Three-Dimensional Algorithm, Finite Element Method, Newtonian Fluid.

\section{INTRODUCTION}

$\Gamma$

he mixing behavior of Newtonian fluid has been investigated in a cylindrical container which is relevant to industrial applications. Industrial problems are very complex to handle, mainly related to the manufacturing of good homogenized mixture. For instance, granular mixing processes [1-2], mixing of toothpaste in industries [3], mixing of dough in food processing industries [4-5], manufacturing of paper by mixing of paper-pulp in paper producing industries [6] and many other mixing processes like paints [7] are very difficult problems in industries. An agitator is an apparatus attached with stirrer to bring fluid into motion by stirring. The use of agitator ensures optimal mixing and homogenization of fluids. The present study investigates this behavior through predicting the flow structure, velocity gradient and pressure differential.
Mixing of non-Newtonian fluid in a cylindrical container with eccentric fixed stirrer placed on the lid of cylindrical vessel without agitator using 2D Finite Element fractional stage predictor-corrector Taylor-Galerkin/Pressurecorrection scheme was demonstrated. The governing equations were used in cylindrical polar coordinate system [3].

Mixing of non-Newtonian fluid in a cylindrical container with three different shapes of stirrer placed in eccentric position on the lid of the cylindrical container using 2D Finite Element fractional step predictor-corrector TaylorGalerkin/Pressure-Correction scheme in cylindrical polar coordinate system was demonstrated [4]. The first problem was that with full-stirrer, second with a horizontal half-

Authors E-Mail: (hameer_akhtar@hotmail.com, ahsanullah.baloch@isra.edu.pk, hkazi@isra.edu.pk,ma.nizamani@isra.edu.pk)

* Department of Computer Science, ISRA University, Hyderabad, Pakistan.

This is an open access article published by Mehran University Research Journal of Engineering and Technology, Jamshoro under the CC by 4.0 International License. 
stirrer and the third with a vertical half-stirrer without agitator. Power consumption and rate of work was observed due to change in the shapes of stirrer.

The numerical simulation in two cylindrical containers: one with single stirrer and the other with two stirrers without agitator has been simulated using fully 3D Finite Element fractional stage predictor-corrector TaylorGalerkin/Pressure-Correction scheme in cylindrical polar coordinate system which requires high computational overload to investigate the mixing behavior of nonNewtonian fluid [8], where the stirrers are located in eccentric position on the lid of the container. Shear-rate and pressure were demonstrated at various speed of stirrer.

The Pseudo 3D Finite Element approach was used to investigate unsteady free surface water profile in channel [9]. The numerical technique was based on fractional stages predictor-corrector Taylor-Galerkin/ Pressure-Correction scheme in Cartesian coordinate system.

In this study, the mixing behavior of Newtonian fluid is investigated in a cylindrical container through single rotating stirrer with agitator, where stirrer is located on the lid of container and positioned concentrically. In CFD (Computational Fluid Dynamics), the flow of Newtonian fluid in cylindrical shaped container is mathematically modelled through non-linear system of partial differential equations, such as conservation of mass and conservation of momentum transport equations in cylindrical polar coordinate system to determine the flow structure, velocity gradient and pressure differential.
FEM (Finite Element Method) has been used as a powerful mathematical tool by many researchers to reduce the complexities in mixing processes, like accurate model of domain boundaries [10], spatial discretization of domain, time discretization of transient flows, use of suitable material properties and boundary conditions [11].

The objective of this study is to develop a Quasi 3D FEA based on fractional stages semi-implicit Taylor-Galerkin/ Pressure-Correction scheme [10] for the rotating mixing flows in cylindrical container through single agitated rotating stirrer and to investigate the effects of agitator on flow structure and pressure gradient. The problem specification is described in section 2 , the details of basic governing equations and numerical scheme used is presented in section 3 and section 4 respectively. The numerical predictions are demonstrated in section 5 and conclusion is described in section 6 .

\section{PROBLEM SPECIFICATION/DEFINITION}

The problem in the present study is to investigate mixing flow of Newtonian fluid, relevant to the pharmaceutical and cosmetic industrial applications. The concentric configuration of a single rotating stirrer with agitator has been adopted, where the stirrer will be located on the lid of the cylindrical container [4]. The mixing is performed between rotation of stirrer with agitator and stationary cylindrical container for numerical simulations. A cylindrical polar coordinate system has been used due to the nature of problem.

In order to present the well posed problem specification for the mixing flows in cylindrical container, it is essential to use the suitable initial and boundary conditions. At initial time, the motionless values have been taken for components of velocity such as:

$\tilde{u}=0$ 
No slip boundary conditions have been imposed at the round disc, solid wall and base of cylindrical container $\left(u_{r}=u_{\theta}=u_{z}=0\right) \cdot u_{r}=u_{z}=0$ and $u_{\theta}=\Omega R$ on the dimensions of stirrer and agitator. The flow of fluid in cylindrical container has been considered to be axi-symmetric; therefore, the numerical simulation has been investigated on the right half of cylindrical container. Mixed boundary conditions have been employed on the axis of symmetry i.e. $u_{r}=u_{z}=0$ and traction free axial $\left(\frac{\partial u_{z}}{\partial r}\right)$ component of velocity has been used. Numerical simulations have been demonstrated through contour plots of stream-function and pressure differentials. The computational domain and finite element mesh concerned for present problem have been displayed in Fig. 1.

For finite element mesh, the total number of nodes, elements and degrees of freedom are 709, 320 and 2322 respectively. The size of each element is $1.4645 \times 10^{-2}$ and computed as radius of the inscribed circle in a triangular element.

\section{GOVERNING EQUATIONS}

The flow of Newtonian fluid in cylindrical container can be mathematically modelled through the system of conservation of mass and conservation of momentum transport equations over domain $\Omega$. For isothermal flow of incompressible fluids in cylindrical polar coordinate system, the component wise continuity equation in the absence of body forces is expressed as:

$\nabla \cdot \tilde{u}=0$

and the momentum transport equation as:

$$
\rho \frac{\partial \tilde{u}}{\partial t}=\nabla \cdot \widetilde{\tilde{u}}-\rho(\tilde{u} \cdot \nabla) \tilde{u}
$$

Whilst,

$\tilde{u}=\left(u_{r}, u_{\theta}, u_{z}\right)$ is the velocity vector field of fluid, $\rho$ is the fluid density, $t$ represents time, $\widetilde{\sigma}$ is the Cauchy stress tensor which can be described as $\widetilde{\widetilde{\sigma}}=-p \partial+\widetilde{\widetilde{T}}$ and $\nabla$ is the spatial differential operator.

Where $p$ is isotropic fluid pressure, Kronecker delta $\delta$ is the unit tensor and $\widetilde{\widetilde{T}}$ is the total stress tensor. For Newtonian fluids, according to Newton's viscous law, the total stress tensor $\widetilde{T}$ is proportional to rate-ofdeformation tensor and can be expressed as:
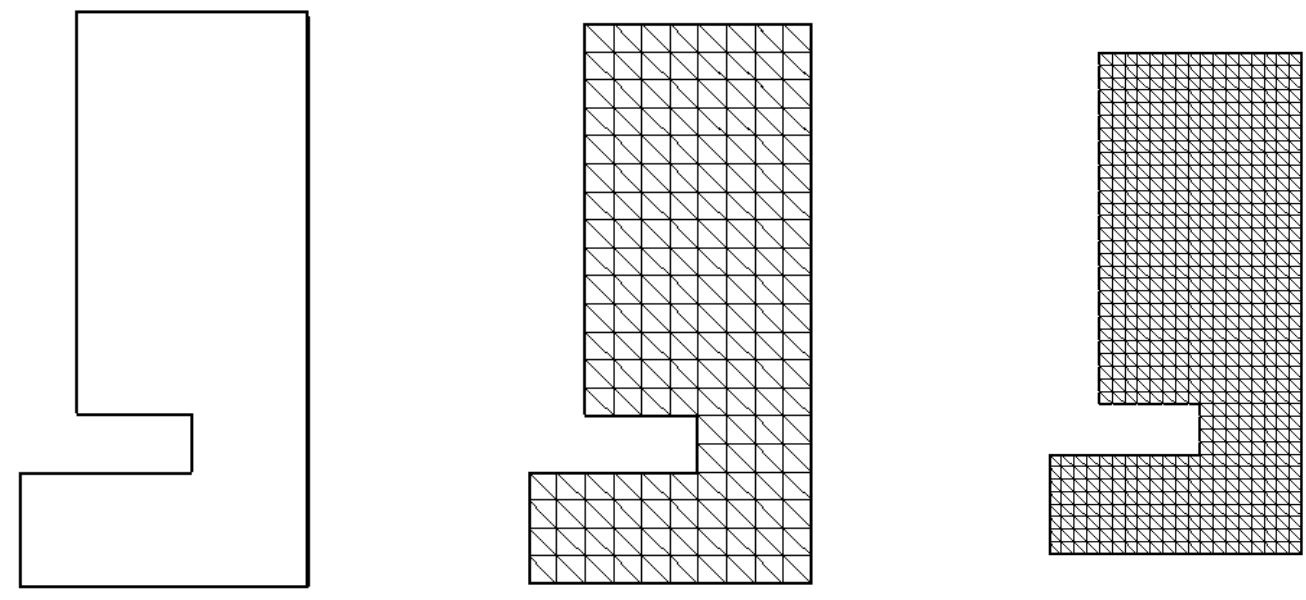

FIG. 1. COMPUTATIONAL DOMAIN OF INTEREST AND FINITE ELEMENT MESH

Mehran University Research Journal of Engineering \& Technology, Volume 38, No. 2, April, 2019 [p-ISSN: 0254-7821, e-ISSN: 2413-7219] 
$\widetilde{\widetilde{T}}=2 \mu \widetilde{\widetilde{d}}$

Where, $\mu$ is the constant fluid viscosity and $\widetilde{\widetilde{d}}$ is the rate-of-deformation tensor which can be defined as $\widetilde{\widetilde{d}}=\frac{1}{2}[\nabla \widetilde{u}+(\nabla \widetilde{u})]$ and $\dagger$ represents transpose operator for matrix transposition. In the absence of body forces and under the proportionality of the total stress tensor to the rate-of-deformation tensor yields the compact form of Navier-Stokes equation as:

$\rho \frac{\partial u_{r}}{\partial t}=\mu \nabla^{2} u-\rho(u . \nabla) u-\nabla p$

For an incompressible flow without body force, the Quasi $3 \mathrm{D}$ component wise system of governing equations without asterisk notation can be expressed nondimensionally as:

$\frac{\partial u_{r}}{\partial r}+\frac{u_{r}}{r}+\frac{\partial u_{r}}{\partial z}=0$

$r: \frac{\partial u_{r}}{\partial r}=\frac{1}{\operatorname{Re}}\left(\frac{\partial^{2} u_{r}}{\partial r^{2}}+\frac{1}{r} \frac{\partial u_{r}}{\partial r}+\frac{\partial^{2} u_{r}}{\partial z^{2}}-\frac{u_{r}}{r^{2}}\right)-\left(u_{r} \frac{\partial u_{r}}{\partial r}+u_{z} \frac{\partial u_{r}}{\partial z}-\frac{u_{\theta}^{2}}{r}\right)-\frac{\partial p}{\partial r}$

(6) $\theta: \frac{\partial u_{\theta}}{\partial t}=\frac{1}{\operatorname{Re}}\left(\frac{\partial^{2} u_{\theta}}{\partial r^{2}}+\frac{1}{r} \frac{\partial u_{\theta}}{\partial r}+\frac{\partial^{2} u_{\theta}}{\partial z^{2}}-\frac{u_{\theta}}{r^{2}}\right)-\left(u_{r} \frac{\partial u_{\theta}}{\partial r}+u_{z} \frac{\partial u_{\theta}}{\partial z}+\frac{u_{r} u_{\theta}}{r}\right)$ (7)

$z: \frac{\partial u_{z}}{\partial t}=\frac{1}{\operatorname{Re}}\left(\frac{\partial^{2} u_{z}}{\partial r^{2}}+\frac{1}{r} \frac{\partial u_{z}}{\partial r}+\frac{\partial^{2} u_{z}}{\partial z^{2}}\right)-\left(u_{r} \frac{\partial u_{z}}{\partial r}+u_{z} \frac{\partial u_{z}}{\partial z}\right)-\frac{\partial p}{\partial z}$

Where, the non-dimensional Reynolds number is defined as:

\section{NUMERICAL SCHEME}

For the computation of the fluid's velocity and pressure, a Quasi 3D time-marching FEA has been developed. This algorithm is based on three different fractional stages semi-implicit Taylor-Galerkin/Pressure-Correction scheme. In this algorithm Taylor series has been expanded up to second order for temporal domain discretization and Galerkin approximation has been used for spatial discretization. For the evaluation of pressure gradient, a pressure-correction scheme has been adopted. The finite element basis functions used in this study are piecewise linear $\left(\Psi_{\mathrm{k}}\right)$ for pressure and piecewise quadratic $\left(\varphi_{\mathrm{a}}\right)$ for velocity. A seven-point Gauss quadrature rule has been used for the evaluation of Galerkin integrals. The fractional stages of Quasi 3D FEA are as follows:

Step-1(a): Compute the velocity component at half time step $n+1 / 2$ from initial velocity

field at time step $t_{n}$.

Step-1(b): Calculate an intermediate non-divergence-free velocity field $u^{*}$ at full time step,

using solutions at levels $n$ and $n+1 / 2$.

Step-2: $\quad$ Calculate pressure difference using $u^{*}$ from step-1(b) via Poisson equation using Choleski method at full time step interval $[n, n+1]$.

Step-3: Compute a divergence-free velocity field $u^{n+1}$ using Jacobi iterative method from pressure difference field of step-2.

\section{NUMERICAL PREDICTIONS AND D I S C USS I O N / N U M E R I C A L RESULTS}

\subsection{Effects of Inertia on Flow Structure}

For mixing of incompressible Newtonian fluid in cylindrical container through single concentric rotating stirrer with agitator, numerical predictions illustrate the flow structures through streamline contours, in Fig. 2, with 
increasing $\operatorname{Re}$ (Reynolds Number) of order O(1) (i.e. $\operatorname{Re}=$ $1,10,25$ and 50) to investigate the homogenization of material. At unit Re, fluid is moving and mixing slowly. Near cylindrical vessel wall fluid is almost stagnant, whereas, below agitator a small recirculation starts to mix the material. It is observed that the $\mathrm{Re}=25$ is found to be a critical value where the fluid starts to be slightly pushed below agitator due to small recirculation. Physically, it shows that the movement of material is less near wall of vessel. As inertia increases, mixing process becomes better due to which the material is pushed towards the vessel wall, stirrer and agitator.

As the inertial level approaches the critical value, i.e. $\mathrm{Re}=76$ and onwards, the recirculation increases below the agitator, this flow phenomenon is displayed in Fig. 3. Vortex centre appears in front of the lower corner of agitator almost at $45^{\circ}$ and becomes stronger as the inertial level reaches at $\mathrm{Re}=500$. This phenomena continues when the inertial level turns out to be equal from $\mathrm{O}(2)$ to $\mathrm{O}(3)$ (i.e. from $\mathrm{Re}=750$ to $\mathrm{Re}=3000$ ) and the vortex eye is moved to the side wall of container as displayed in Fig. 4 which shows that material is shift towards wall and creates space at centre. The increase in inertia again pushes material in vacuum. The material collides with agitator and this process is continued till homogenised mixture is obtained.

\subsection{Effects of Inertia on Pressure}

The fluid flow becomes asymmetric and instability starts after $\mathrm{Re}=3000$, also vortex centre changes its location.
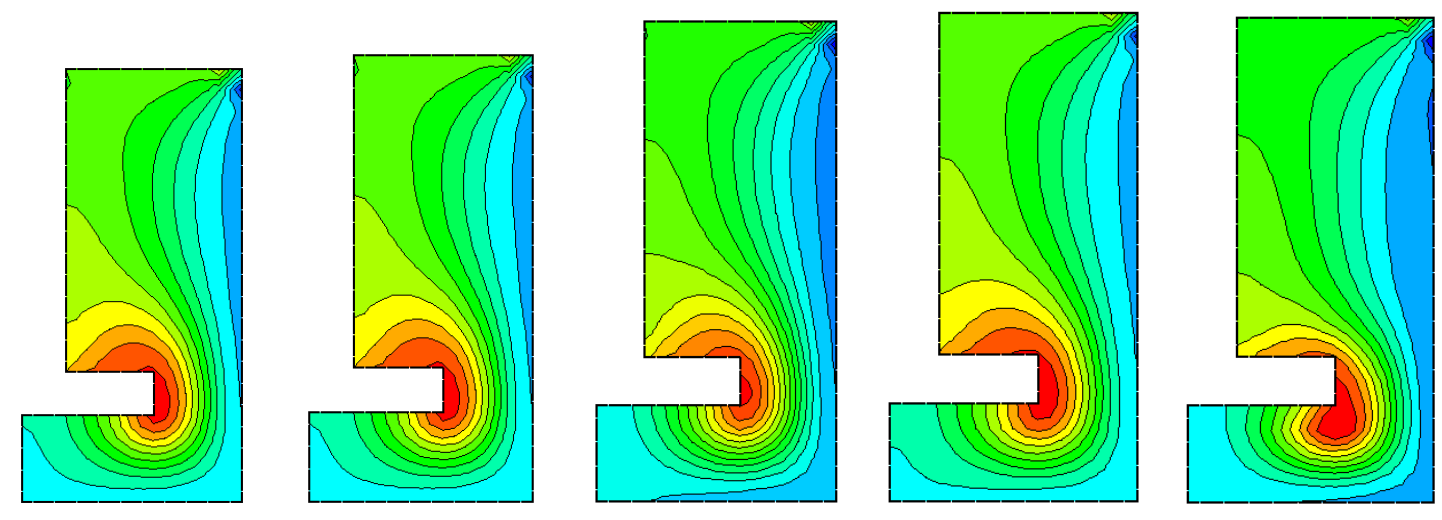

FIG. 2. STREAMLINE CONTOURS OF ROTATING STIRRER WITH AGITATOR WITH INCREASING INERTIA FROM $R e=1,10,20,25 A N D 50$
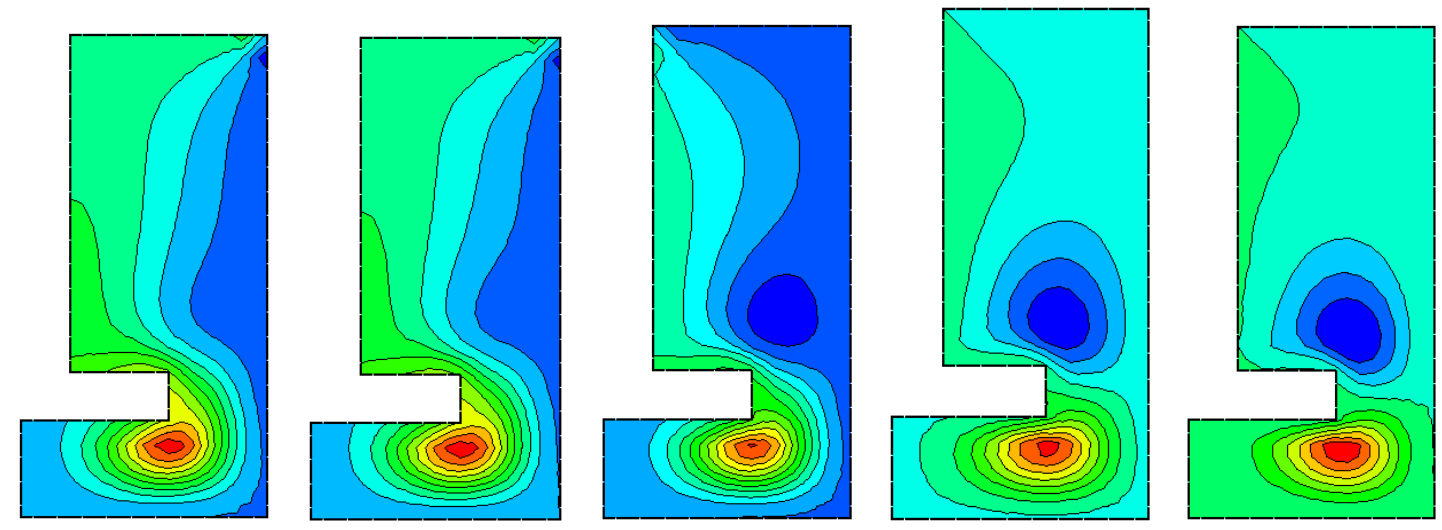

FIG. 3. STREAMLINE CONTOURS OF ROTATING STIRRER WITH AGITATOR WITH INCREASING INERTIA FROM $R e=76,80,100,200 A N D 500$

Mehran University Research Journal of Engineering \& Technology, Volume 38, No. 2, April, 2019 [p-ISSN: 0254-7821, e-ISSN: 2413-7219] 
Due to this instability, flow changes its state from laminar to turbulent which is the limitation of present research study. For vortex intensity computed from stream function tabulated as maximum and minimum stream function, the values of pressure and relative pressure drop is also tabulated in Table 1.

The third main aspect of this chapter is to compute the Pressure gradient. The isobars of pressure differentials with increasing levels of inertia of order from $\mathrm{O}(1)$ to $\mathrm{O}(3)$ are displayed in set of Figs. 5-7.
It is observed that the pressure increases slightly at low values of Re. But as the values of Re increases, the pressure drops rapidly and appears to be horizontal asymptotic in nature. The pressure drop creates void space at centre and shifts material towards wall of vessel.

The RPD (Relative Pressure Drop) of fluid in cylindrical container is calculated by the following formula:

$$
R P D=\frac{\left|P_{\max }-P_{\min }\right| * \mathrm{Re}}{\left|P_{\min }\right|_{\mathrm{Re}=1}}
$$
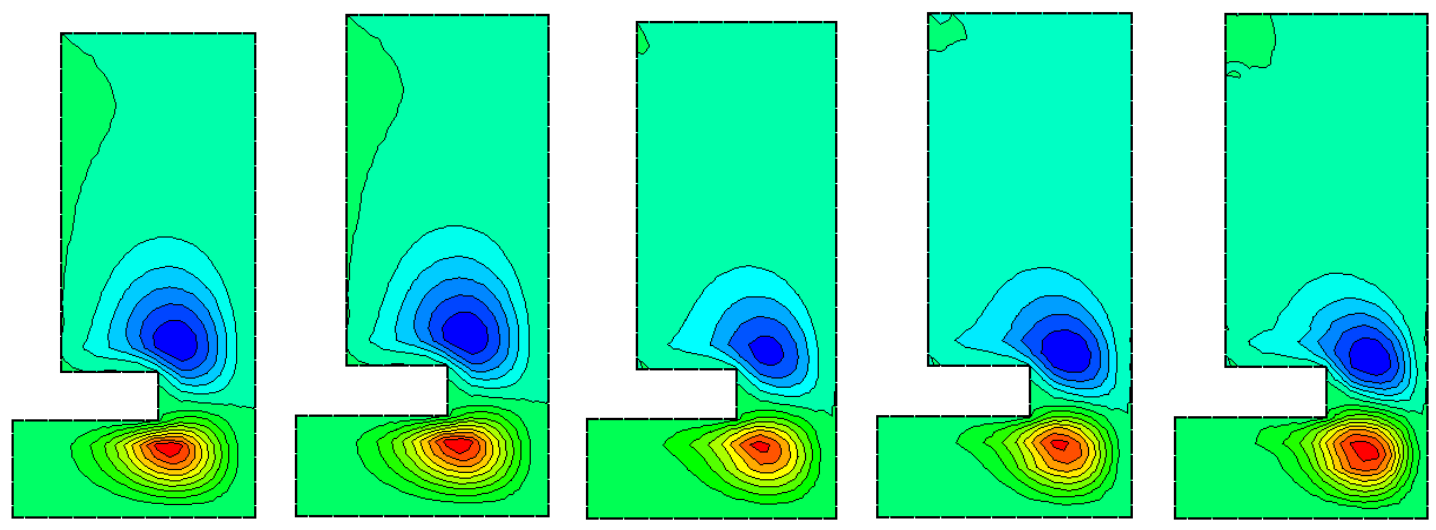

FIG. 4. STREAMLINE CONTOURS OF ROTATING STIRRER WITH AGITATOR WITH INCREASING INERTIA FROM Re = 750, 1000, 1500, 2000 AND 3000

TABLE 1. VARIOUS VALUES OF STREAM FUNCTION, SPEED, PRESSURE AND RELATIVE PRESSURE DROP FOR ROTATING STIRRER WITH AGITATOR IN CYLINDRICAL CONTAINER

\begin{tabular}{|c|c|c|c|c|c|c|c|}
\hline \multirow{2}{*}{$\operatorname{Re}$} & \multicolumn{2}{|c|}{ Stream Function } & \multicolumn{2}{|c|}{ Speed } & \multicolumn{2}{|c|}{ Pressure } & \multirow{2}{*}{$\begin{array}{c}\text { Relative Pressure } \\
\text { Drop }\end{array}$} \\
\hline & Minimum & Maximum & Minimum & Maximum & Minimum & Maximum & \\
\hline 1 & -0.0321 & 0.02963 & 0 & 1.0590 & -467.82 & $-4.6720 \times 10-18$ & 1.0000 \\
\hline 10 & -0.0321 & 0.02963 & 0 & 0.1059 & -4.6788 & $-4.6730 \times 10-20$ & 0.0100 \\
\hline 20 & -0.0016 & 0.00149 & 0 & 0.0529 & -1.1697 & $-1.1683 \times 10-20$ & $2.5000 \times 10^{-3}$ \\
\hline 25 & -0.0013 & 0.00120 & 0 & 0.0424 & -0.7486 & $-7.4772 \times 10-21$ & $1.6000 \times 10^{-3}$ \\
\hline 50 & -0.00062 & 0.00072 & 0 & 0.0212 & -0.1871 & $-1.8693 \times 10-21$ & $4.0000 \times 10^{-4}$ \\
\hline 76 & -0.00039 & 0.00078 & 0 & 0.0139 & -0.0840 & $-8.0908 \times 10-22$ & $1.7900 \times 10^{-4}$ \\
\hline 80 & -0.00037 & 0.00079 & 0 & 0.0132 & -0.0770 & $-7.3020 \times 10-22$ & $1.4997 \times 10$ \\
\hline 100 & -0.00027 & 0.00092 & 0 & 0.0201 & -0.0452 & 0.0000 & $9.6572 \times 10^{-5}$ \\
\hline 200 & -0.00077 & 0.00149 & 0 & 0.0328 & -0.0196 & 0.0000 & $4.1874 \times 10^{-5}$ \\
\hline 500 & -0.00206 & 0.00344 & 0 & 0.0814 & -0.0125 & 0.0024 & $3.1879 \times 10^{-5}$ \\
\hline 750 & -0.00290 & 0.00489 & 0 & 0.1148 & -0.0119 & 0.0031 & $3.2132 \times 10^{-5}$ \\
\hline 1000 & -0.00290 & 0.00489 & 0 & 0.1364 & -0.0118 & 0.0033 & $3.2364 \times 10^{-5}$ \\
\hline 1500 & -0.00437 & 0.00754 & 0 & 0.1540 & -0.0123 & 0.0034 & $3.3397 \times 10^{-5}$ \\
\hline 2000 & -0.00496 & 0.00833 & 0 & 0.1629 & -0.0130 & 0.0034 & $3.5056 \times 10^{-5}$ \\
\hline 3000 & -0.00583 & 0.00938 & 0 & 0.2039 & -0.0145 & 0.0036 & $3.8878 \times 10^{-5}$ \\
\hline
\end{tabular}

Mehran University Research Journal of Engineering \& Technology, Volume 38, No. 2, April, 2019 [p-ISSN: 0254-7821, e-ISSN: 2413-7219] 
Where $P_{\max }$ and $P_{\min }$ are used for maximum and minimum values of pressure respectively. The graph of relative pressure drop against increasing Re is shown in Fig. 8. It is observed that the magnitude of pressure drop decreases rapidly as value of Reincreases. Therefore, the curve is horizontal asymptotic in nature.
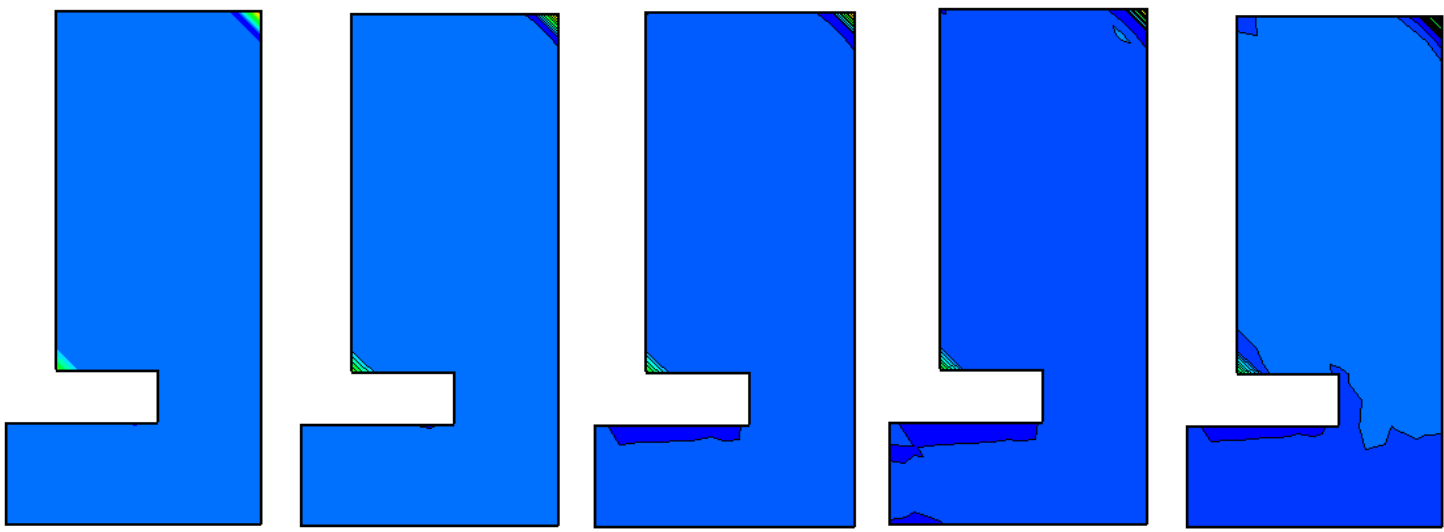

FIG. 5. PRESSURE ISOBARS OF ROTATING STIRRER WITH AGITATOR WITH INCREASING INERTIA FROM $R e=1,10,20,25$ AND 50
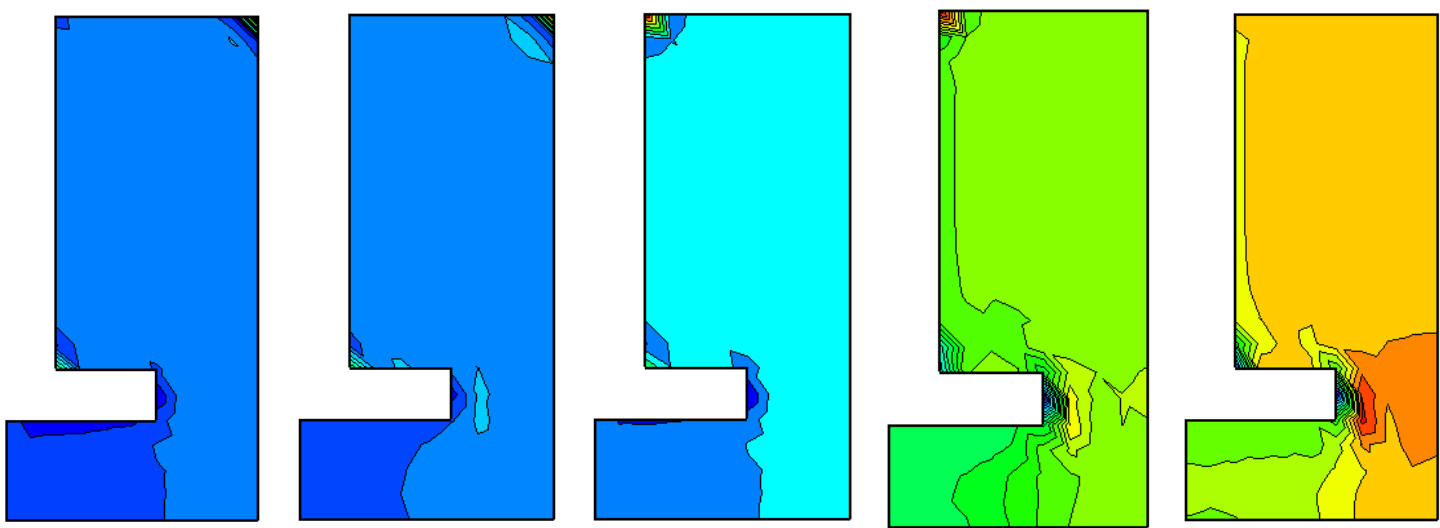

FIG. 6. PRESSURE ISOBARS OF ROTATING STIRRER WITH AGITATOR WITH INCREASING INERTIA FROM $R e=76,80,100,200 A N D 500$
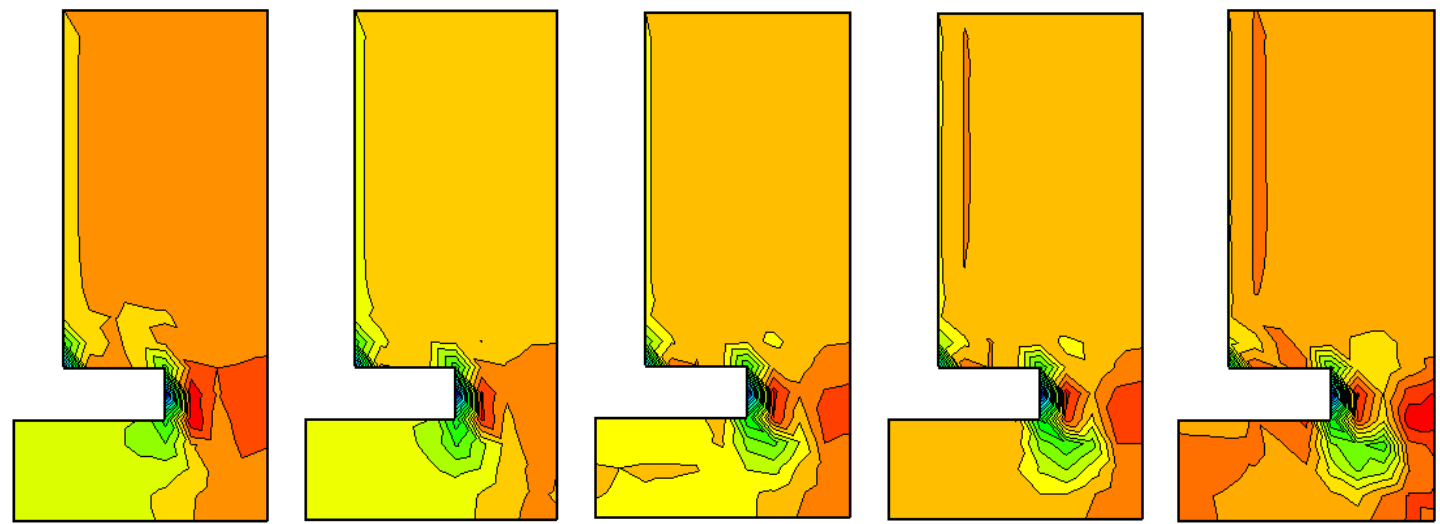

FIG. 7. PRESSURE ISOBARS OF ROTATING STIRRER WITH AGITATOR WITH INCREASING INERTIA FROM $R e=750,1000,1500,2000 A N D 3000$ 


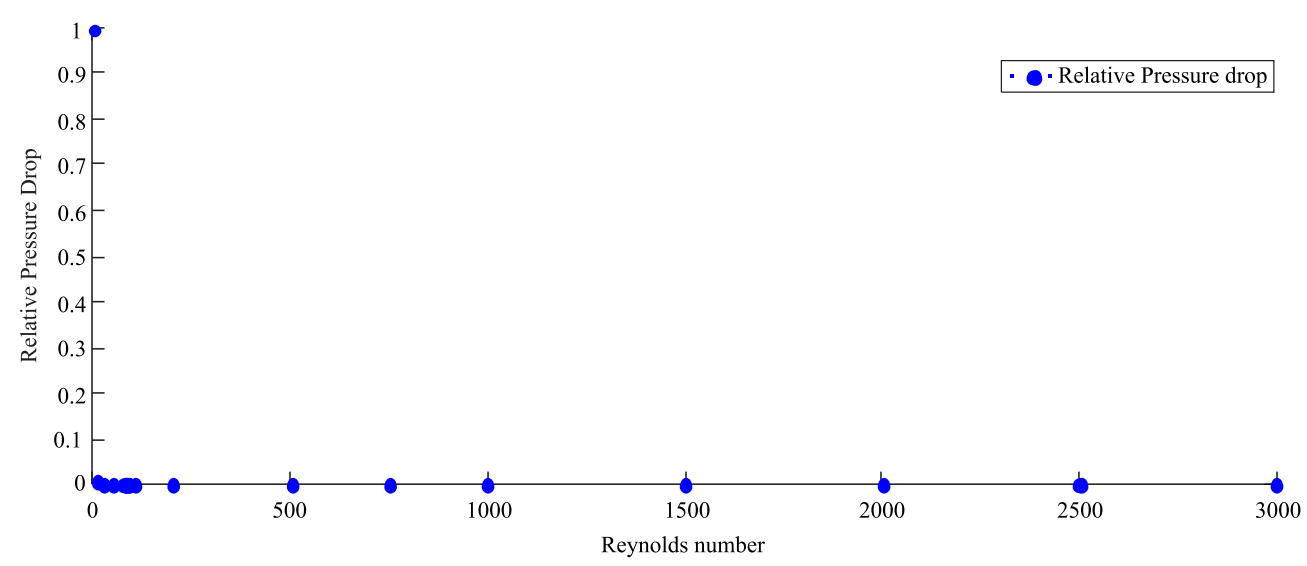

FIG. 8. RELATIVE PRESSURE DROP WITH INCREASING REYNOLDS NUMBER

\section{CONCLUSION}

A Quasi 3D Taylor-Galerkin/Pressure-Correction FEA has been successfully developed for the mixing flows of Newtonian fluid in cylindrical container through single rotating agitated stirrer. The stirrer is concentrically located on lid of vessel. It is clearly displayed that with increasing inertia, recirculation of fluid increases in the vicinity of agitator which is due to the presence of agitator. The recirculation creates vacuum at centre. The increase in inertia again pushes material in empty space at critical values. The vortex improvement at various inertial levels has been focused. The relative pressure drop is almost horizontal asymptotic in nature at increasing inertial levels which shows that the void space is created at centre of vessel. The material is pushed again in void space. This process continues till the homogenized mixture is obtained.

\section{ACKNOWLEDGEMENT}

Authors are greatly acknowledged to the ISRA University, Hyderabad, Pakistan, for providing the facility to conduct this research study.

\section{REFERENCES}

[1] Portillo, P.M., Muzzio, F.J., and Ierapetritou, M.G., "Hybrid DEM Compartment Modelling Approach for Granular Mixing", The American Institute of Chemical Engineers, 2007.

[2] Saeed, S., Ein-Mozaffari, F., and Upreti, S.R., "Using Computational Fluid Dynamics Modelling and Ultrasonic Doppler Velocimetry to Study Pulp Suspension Mixing", Industrial and Engineering Chemistry Research, 2007.
[3] Bhatti, I., Baloch, A., and Qureshi, K.,'Finite Element Modelling of Rotating Mixing of Toothpaste", International Journal of Mathematical and Computer Sciences, 2010.

[4] Baloch, A., and Webster, M.F.,"Distributed Parallel Computation for Complex Rotational Flows of NonNewtonian Fluids", International Journal for Numerical Methods in Fluids, 2003.

[ 5] Binding, D.M., Couch, M.A., Sujatha, K.S., and Webster, M.F., "Experimental and Numerical Simulation of Dough Kneading in Filled Geometries", Journal of Food Engineering, 2003.

[6] Jung, H., and Pauly, D.,"Water in the Pulp and Paper Industry", Reference Module in Earth Systems and Environmental Sciences, from Treatise on Water Science, 2014.

[7] Wijewardane, S., and Goswami, D.Y.,"A Review on Surface Control of Thermal Radiation by Paints and Coatings for New Energy Applications", Renewable and Sustainable Energy Reviews, 2012.

[8] Sujatha, K.S., Ding, D., and Webster, M.F.,"Modelling Three-Dimensional Mixing Flows in Cylindrical Vessels", Proceedings of Computational Fluid Dynamics Conference, Swansea, UK, 2001.

[9] Mahessar, A.A., Baloch, A., and Qureshi, A.L., "Pseudo Three-Dimensional Finite Element Model for Unsteady Free Surface Flow", Jokull Journal, 2014.

[10] Townsend, P., and Webster, M.F.,"An Algorithm for the Three-Dimensional Transient Simulation of NonNewtonian Fluid Flows", Pande, G.N., and Middleton, J., (Editors), "Transient/Dynamic Analysis and Constitutive Laws for Engineering Materials", Proceedings of Numerical Mathematics Engineering, Theory and Applications, Nijhoff, Kluwer, Dordrecht, 1987.

[11] Hawken, D.M., Tamaddon-Jahromi, H.R., Townsend, P., and Webster, M.F., "A Taylor-Galerkin-Based Algorithm for Viscous Incompressible Flow", International Journal of Numerical Methods in Fluids, 1990. 